\title{
Alterations in the blood-spinal cord barrier in TDP-43 conditional knockout mice
}

Running title: BSCB changes in TDP-43 knockout mice

\author{
Shoichi Sasaki, $\mathrm{MD}, \mathrm{PhD}^{\left.1^{*}\right)}$, Yohei Iguchi, $\mathrm{MD}, \mathrm{PhD}^{2)}$, \\ Masahisa Katsuno, $\mathrm{MD}, \mathrm{PhD}^{2)}$, and Gen Sobue, $\mathrm{MD}, \mathrm{PhD}^{2)}$
}

${ }^{1}$ Department of Neurology, Tokyo Women's Medical University, Tokyo, Japan

2 Department of Neurology, Nagoya University Graduate School of Medicine, Nagoya, Japan

* Corresponding author: Shoichi Sasaki, M.D. PhD

Department of Neurology,

Tokyo Women's Medical University,

8-1 Kawada-cho, Shinjuku-ku,

Tokyo 162-8666,

Japan,

Tel: 81-3-3353-8111 ext. 39232,

Fax: 81-3-5269-7324,

E-mail: ssasaki@nij.twmu.ac.jp 


\begin{abstract}
We investigated whether the loss of motor neuron-specific TDP-43 protein causes any change in the blood-spinal cord barrier (BSCB) in the spinal cord of TDP-43 conditional knockout (TDP CKO) mice. The TDP CKO mice were divided into four groups: early presymptomatic, late presymptomatic, early symptomatic, and late symptomatic stages. The spinal cords were pathologically examined. TDP CKO mice showed the activation of MAC-2 (macrophages/microglia) and fibrinogen exclusively in the anterior horn from the early symptomatic through the late symptomatic stages. Immunohistochemical and Western blot analyses detected no reduction in tight junction proteins in TDP CKO mice as compared with age-matched wild-type mice at any stage. Electron-microscopically, TDP CKO mice showed vacuoles in the cytoplasm of most endothelial cells at the early symptomatic stage. The endothelium occasionally exhibited swollen cytoplasm by edematous fluid with the intact tight junction. The cytoplasm of the pericytes was relatively well preserved in contrast to the endothelial disruption. Extravascular or perivascular spaces were frequently edematous and vacuolated. At the other stages, the BSCB was well preserved as in the controls. Thus, the temporary and reversible breakdown of the BSCB with leakage or increased permeability at the early symptomatic stage observed in this study could be a direct pathogenic consequence of the loss of TDP-43 protein, and the temporal impairment of $\mathrm{BSCB}$, in turn, might contribute to the motor neuron degeneration in TDP CKO mice.
\end{abstract}

Keywords: amyotrophic lateral sclerosis, TDP-43 knockout mice, blood-spinal cord barrier, tight junction proteins, fibrinogen, ultrastructure 


\section{Introduction}

The blood-spinal cord barrier (BSCB) of the spinal cord capillary consists of non-fenestrated endothelial cells with tight junctions, basal lamina, pericytes, and astrocyte feet processes [1]. The BSCB plays a protective and regulatory role for the spinal cord parenchyma to maintain and control the homeostasis of the central nervous system. The factors influencing the integrity of the BSCB stem from neurons and microglia as well as pericytes and astrocytes.

It was not until recently that microvascular competence in the brain and spinal cord, which suggests impairment of blood brain barrier (BBB) and BSCB integrity in animal models of ALS and in ALS patients [2], attracted special attention. However, the role of BSCB including capillaries in the pathogenesis of ALS is not still clear. We recently developed motor neuron-specific transactive response DNA-binding protein 43 (TDP-43) conditional knockout (TDP CKO) mice [3]. The mice show an age-dependent progressive motor dysfunction accompanied by neuropathological changes that are common to sporadic ALS, suggesting that TDP-43 plays an essential role in the long term maintenance of motor neurons and that the loss-of-function of this protein contributes to the pathogenesis of ALS. To elucidate the neurovascular system, we neuropathologically focused on the BSCB in TDP CKO mice to determine whether the loss of motor neuron-specific TDP-43 causes any change in the BSCB.

\section{Materials and methods}

All animal experiments were performed in accord with the National Institutes of Health Guide for the Care and Use of Laboratory Animals and under the approval of the Nagoya University Animal Experiment Committee (Nagoya, Japan).

\section{Light microscopy study}

The TDP CKO mice were divided into four groups: early presymptomatic (aged 20 
wks), late presymptomatic (36 wks), early symptomatic (50 wks), and late symptomatic stages (100 wks) $(\mathrm{n}=2$, respectively). Age-matched wild type mice served as age-matched control littermates in each group $(n=2)$. Age-matched control and TDP CKO mice were deeply anesthetized with an interperitoneal injection of medetomidine $(0.3 \mathrm{mg} / \mathrm{kg})$, midazolam $(4 \mathrm{mg} / \mathrm{kg})$ and butorphanol $(5 \mathrm{mg} / \mathrm{kg})$. The mice were then perfused with $20 \mathrm{~mL}$ of a $4 \%$ paraformaldehyde fixative in phosphate buffer (Wako, Osaka, Japan) through the left cardiac ventricle to the spinal cord, for both the control and TDP CKO mice. Tissues postfixed overnight in 10\% phosphate-buffered formalin were then processed for paraffin embedding. Paraffin-embedded tissues $(3-\mu \mathrm{m}$ thick) were stained with hematoxyline and eosin (H\&E).

\section{Immunohistochemical study}

Paraffin-embedded tissues stained with H\&E of the cervical spinal cords of TDP CKO mice and age-matched wild-type control mice, were immunostained with anti-mouse MAC-2 (Galectin-3) (macrophages/microglia) monoclonal antibody (1:500;

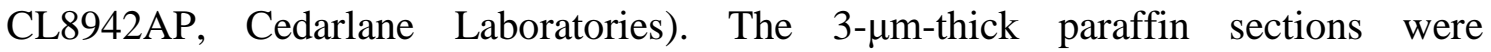
deparaffinized, rehydrated, quenched for 10 min at $4{ }^{\circ} \mathrm{C}$ in $3 \% \mathrm{H}_{2} \mathrm{O}_{2}$, rinsed in $\mathrm{pH} 7.6$ phosphate-buffered saline (PBS), pretreated for $20 \mathrm{~min}$ at room temperature with $5 \%$ skim milk in PBS and then incubated overnight at $4^{\circ} \mathrm{C}$ with the primary antibodies. For the antibody against MAC-2, immunoreaction was visualized by the polymer immunocomplex (PIC) method using the ENVISION system (Dako, k5027). Sections of the lumbar spinal cords of additional TDP CKO mice (20, 50, and 100 wks, $n=3$, respectively) and age-matched wild-type control mice (20, 50, and 100 wks, n=3, respectively) to be stained with anti-fibrinogen and anti-claudin-5 antibodies were boiled in $10 \mathrm{mM}$ citrate buffer for $15 \mathrm{~min}$. The primary antibodies and their dilutions were as follows: fibrinogen (1:1000; sc-33580, Santa Cruz) and claudin-5 (1:2000; 
ab53765, Abcam). Primary antibody binding was probed with a secondary antibody labeled with a polymer as part of the ENVISION system containing horseradish peroxidase (Dako, k5027). All sections were counterstained with hematoxylin. Sections from which the primary antibodies were omitted served as negative reaction controls.

\section{Western blotting}

The ventral half of the lumbar spinal cord from the wild-type and TDP CKO mice at the ages of 20,50 , and 70 wks $(n=3)$ were homogenized in buffer containing CelLytic ${ }^{\mathrm{TM}}$ M Mammalian Cell Lysis/Extraction Reagent (Sigma, St. Louis, MO) with 1 $\mathrm{mM}$ phenylmethylsulfonyl fluoride (PMSF) and $6 \mu \mathrm{g} / \mathrm{mL}$ aprotinin and then centrifuged at $2,500 \times \mathrm{g}$ for $15 \mathrm{~min}$. Equal amounts $(50 \mu \mathrm{g})$ of protein were separated by $5 \%-20 \%$ sodium dodecyl sulfate/polyacrylamide gel electrophoresis (SDS/PAGE) and transferred to Hybond-P membranes (GE Healthcare, Piscataway, NJ). Primary antibody binding was probed with horseradish peroxidase-conjugated secondary antibodies at a dilution of 1:5000, and bands were detected by using an enhanced chemiluminescence system (ECL Prime; GE Healthcare). An LAS-3000 imaging system (Fujifilm, Tokyo) was used to produce digital images. The membranes were reprobed with an anti-glyceraldehyde-3-phosphate dehydrogenase (GAPDH) antibody for normalization. The primary antibodies used were as follows: claudin-5 (1:1000, ab53765, Abcam), occludin (1:500, ab31721, Abcam), ZO-1 (1:100, ab59720, Abcam), and GAPDH (1:5000, MAB374, Millipore, Bedford, MA).

\section{Electron-microscopy study}

We studied the lumbar spinal cords in eight TDP CKO mice (20, 36, 50, and 100 wks, $\mathrm{n}=2$, respectively) and eight wild-type mice served as age-matched control littermates (20, 36, 50, and 100 wks, $n=2$, respectively). Under deep anesthesia with isoflurane, mice were transcardially perfused with $3 \%$ paraformaldehyde and $1 \%$ 
glutaraldehyde in PBS. The spinal cords were removed and fixed in a solution $(0.1 \mathrm{M}$ PBS, 3.5\% paraformaldehyde, $0.5 \%$ glutaraldehyde). After fixation, the spinal cords were immersed in a solution $(0.2 \mathrm{M}$ cacodylic acid $5 \mathrm{~mL}, 10 \%$ paraformaldehyde $2 \mathrm{~mL}$, $25 \%$ glutaraldehyde $1 \mathrm{~mL}$, distilled water $2 \mathrm{~mL}$ ) for $12 \mathrm{~h}$. Each cervical spinal cord was then sectioned transversely, postfixed in $1 \%$ osmium tetroxide for several hours, dehydrated, and embedded in epoxy resin. Each block was cut into serial semi-thin sections (approx. 1- $\mu \mathrm{m}$ thick) and stained with toluidine blue. Appropriate portions of the hemi-sections of the cervical spinal cords including the anterior and posterior horns were cut into ultrathin sections, which were then stained with uranyl acetate and lead citrate for electron microscopy.

\section{Results}

\section{Light microscopic analysis}

The average number of motor neurons in the 100-wk-old TDP CKO mice did not differ from that in the wild-type littermates, although TDP-43 was knocked-out in $~ 50 \%$ of the motor neurons in the TDP CKO mice. The average size of the TDP-43-lacking spinal motor neurons in the TDP CKO mice was decreased at 100 wks of age compared to the TDP-43-positive motor neurons and motor neurons in the control littermates [3].

\section{Immunohistochemical analysis}

Activation of macrophages/microglia was not observed anywhere in the spinal cord in the TDP CKO mice at the ages of 20 and 36 wks. However, at the age of 50 wks, the spinal cord, exclusively in the anterior horn, was positively immunostained for MAC-2, indicating definite activation of macrophages/microglia (Fig. 1a), which persisted until the late symptomatic stage (100 wks). In the controls, no increased immunoreactivity of MAC-2 was exhibited anywhere on the spinal cord including the anterior horn at any age. Fibrinogen-positive immuoreactivity was largely confined to the capillaries in the 
anterior horns and not spreading far into the parenchyma at the age of 50 wks (Fig. 1b), which persisted until the late symptomatic stage (100 wks). Fibrinogen immunoreactivity was neither observed in TDP CKO mice at the ages of 20 wks nor in age-matched wild-type mice. No difference of immunoreactivity of claudin-5 was recognized anywhere in the spinal cord between TDP CKO mice and age-matched wild type mice at the ages of 20,50, and $100 \mathrm{wks})$.

\section{Western blot analysis}

There was no detectable difference in the expression levels of claudin-5, occludin, or ZO-1 in the spinal cords of the wild-type mice and those of the TDP CKO mice at the ages of 20, 50 and 70 wks (Fig. 2).

\section{Electron microscopy analysis of the blood-spinal cord barrier Controls}

In the anterior horn of the spinal cord, the transverse sections of the capillaries consisted of endothelial cells and pericytes surrounded by two basal laminae and astrocytic foot processes (Fig. 3a). Each endothelial cell and pericyte was encircled by a basal lamina, and the endothelial cell and the pericyte were separated by a basal lamina in between. They were also surrounded farther outside by another basal lamina. The endothelial cells of the capillaries were characterized by tight junctions and relatively thin cytoplasm (Fig. 3b). The majority of the capillaries were surrounded by a narrow perivascular space without connective tissue. In some instances the perivascular space was virtually obliterated and the two laminae formed a single homogenous structure. In the posterior horn of the spinal cord, the structure of the capillaries was the same as that in the anterior horn.

\section{TDP CKO mice}




\section{Early presymptomatic stage (20 wks)}

In the anterior horn of the spinal cord, the BSCB was normal-looking and showed the same structure as those in the age-matched wild-type control littermates. In the posterior horn of the spinal cord, the structure of the BSCB was normal.

\section{Late presymptomatic stage (36 wks)}

The BSCB of the anterior and posterior horns in the spinal cord appeared intact, showing normal endothelium including tight junctions, pericytes, the basal lamina and perivascular space. The endothelium only occasionally exhibited the protrusion of a part of the cytoplasm into the lumen of the blood vessel. Mitochondria and rough endoplasmic reticulum were well preserved in the cytoplasm of the endothelial cells and pericytes.

\section{Early symptomatic stage (50 wks)}

The cytoplasm and nuclei of most of the endothelial cells were degenerated, showing electron-dense configurations. The cytoplasm of the endothelial cells frequently showed vacuoles from small to large in size and single to multiple in number (Fig. 3c). The basement membrane directly attached to the vacuoles of cytoplasm of the endothelium showed thinness or a defect. The endothelium exhibited cytoplasm that was focally or diffusely swollen with edematous fluids, detachment of the cytoplasm, and the prominent protrusion of some parts of the cytoplasm into the lumen of the blood vessel, although the tight junction appeared relatively intact (Fig. 3d). The mitochondria of the endothelial cells were occasionally swollen and vacuolated. Pericytes frequently proliferated and circumvented around the whole blood vessel (Fig. 3d), and the cytoplasm was relatively well preserved, but occasionally degenerated, containing disorganized materials. Mitochondria in the cytoplasm of pericytes were also occasionally swollen and vacuolated. Extravascular spaces (end-feet of the astrocyte) of 
the capillaries were frequently edematous and vacuolated, occasionally accompanied by macrophages. In the perivascular space between the double basement membranes of the capillary, multilayered sheet-like expansions composed of lamellae of flattened processes of astrocytes were not uncommonly observed. The basal lamina of the astrocytic foot processes was occasionally split into a few basal laminae containing degenerated organellae. In the posterior horn of the spinal cord, the BSCB was well preserved and showed normal structures.

\section{Late symptomatic stage (100 wks)}

The BSCB of the anterior horn in the spinal cord was well preserved, showing normal-appearing endothelia, pericytes, basal laminae and perivascular spaces, which were the same structure as those of the early and late presymptomatic stages and of the age-matched wild-type control mice. The pathological changes of the BSCB observed in the anterior horns at the early symptomatic stage had disappeared and the BSCB appeared to be normal-looking. The BSCB in the posterior horns were well preserved.

\section{Discussion}

There is increasing evidence that the immune system [4] and increased permeability of the BSCB [5] may play fundamental roles in the pathogenesis or progression of pathological changes of the spinal cord in ALS patients and in $\mathrm{Cu} / \mathrm{Zn}$ superoxide dismutase (SOD1) mutant mice modeling ALS. In the cerebrospinal fluid (CSF) of patients with ALS, increased levels of albumin, IgG, and C3c [6,7] have been detected. In the brain and spinal cord of both patients with ALS and animal models of ALS, deposits of $\operatorname{IgG}$ [8.9], the presence of T cell lymphocytes in the affected regions of the spinal cord [4, 10], complements C3 and C4 [8], and lymphocytes, microglia/macrophage and dendritic cells $[10,11]$ have been observed. Moreover, various BSCB leakage markers have been reported in SOD1 mutant animals: Evans 
blue leakage around the spinal cord microvessels at the symptomatic stage $[12,13]$; down-regulation of glucose transporter isoform 1 (Glut-1) and CD146 expressions in the endothelial cells of BSCB [12]; and IgG and hemosiderin leakage [9,13]. The leakage of immunoglobulins that interact with motor neuron antigens can initiate an autoimmune response, which in turn can result in demyelination, disruption of neuronal transmissions, and neuronal cell death. Hemoglobin released from extravasated red blood cells contributes to direct toxicity to neurons and neuronal loss [14]. In addition, at the electron microscopic level, mitochondrial abnormalities in endothelial cells, vacuolated cytoplasm of the endothelial cells, swollen astrocytes and extensive extracellular edema have been observed in SOD1 (G93A) mutant mice at early and late stages $[13,15]$, and focal accumulation of edema between the capillary vessel wall and motor neurons have been reported in SOD1 mutant mice at ages before motor neuron loss [9]. The BSCB disruption with erythrocyte extravasation and pericyte loss was recently reported to be present in the spinal cord of patients with ALS [16]. All these findings suggest that the BSCB is injured, disrupted and implicated in the pathogenesis of ALS patients and SOD1 mutant models of ALS.

TDP-43 is an established pathological hallmark of ALS. Although the loss-of-function of TDP-43 protein seems to affect the pathogenesis of ALS, it remains unknown whether the loss of motor neuron-specific TDP-43 protein is associated with the changes in the BSCB. In the present study, we observed markedly activated immunoreactivity of fibrinogen at the early symptomatic stage (aged 50 wks) in the capillaries of the anterior horns in the TDP CKO mice. This indicates the possibility of the BSCB disruption, because fibrinogen is a well-documented marker of BSCB leakage in both human autopsy tissues [17] and animal model systems [18]. Moreover, electron-microscopic study showed that vacuolar formation and edematous swelling of the cytoplasm in the endothelium and edematous dilatation of the extravascular or perivascular spaces occurred temporarily at the early symptomatic stage in the TDP 
CKO mice, which is consistent with the period of the development of initial symptoms, and with the appearance of macrophages/microglia in this study and astrogliosis by GFAP immunohistochemistry [3] in the anterior horns. At this stage, neither the number of motor neurons in the TDP CKO mice nor the average size of the TDP-43-lacking spinal motor neurons differed from those in the wild-type littermates, although TDP-43 was knocked-out in $\sim 50 \%$ of the motor neurons in the TDP CKO mice [3]. These findings indicate that the alterations of the BSCB could be an early pathological change prior to motor neuron degeneration due to a consequence of direct toxicity of knockdown of motor neuron-specific TDP-43, and these alterations may be associated with the induction of motor neuron degeneration in the TDP CKO mice.

Irrespective of the striking alterations of endothelia, the tight junctions in the TDP CKO mice were well preserved. The involvement of the tight junctions of the endothelium in ALS has also been a topic of debate. The levels of tight junction proteins, claudin-5, ZO-1 and occludin between endothelial cells are reduced in SOD1 mutant mice, indicating a disruption of the BSCB [9]. Moreover, the impairment of BSCB permeability is associated with decreased mRNA expression of tight junction proteins (ZO-1 and occludin), and the basement membrane protein (agrin) in SOD1(G93A) rats [13], which is consistent with a decreased mRNA expression of a tight junction protein (ZO-1) in the lumbar spinal cords of patients with sporadic ALS, although no differences were observed in claudin-5 mRNA expression between sporadic or familial ALS and non-neurodegenerative disease controls [19]. In contrast, on the fine structural level, capillary endothelial cell tight junctions appear to remain intact in early and late symptomatic stages in SOD1 (G93A) mutant mice, despite the presence of progressive extracellular edema in neural tissue $[13,15]$. In the present study, the tight junction proteins such as claudin-5 in the TDP CKO mice were not reduced immunohistochemically at any age as compared with those of the age-matched wild-type controls. Among the tight junction proteins, claudin-5 has been reported to 
occur in the $\mathrm{BBB}$, which seems to be constitutively expressed in all endothelial cells [20]. Also, a western blotting analysis showed no significant reduction in the expression levels of claudin-5, occludin or ZO-1 in the TDP CKO mice compared to the controls at any age. These tight junctions were shown by electron- microscopy to be well preserved through all stages. This may be linked to the finding that pericytes were relatively well preserved in contrast to the endothelial disruption, for pericytes have been shown to control the expression of tight junction proteins and play a key role in regulating the BSCB integrity in the spinal cord and degeneration of pericytes leads to motor neuron degenerative changes [21].

The pathological changes of the endothelia, pericytes, and extravascular or perivascular spaces observed in this study are intriguing in that they temporarily appeared exactly at the phase of the initiation of neurological symptoms and recovered almost completely as time passed. As for the transient breakdown of the BSCB, there have been some reports, which could explain the pathomechanisms: breakdown of the BBB occurs focally and transiently over a protracted period in association with reactive mechanisms that direct repair and growth in Alzheimer's disease [22]; mutant SOD1 microglia isolated from ALS mice at disease onset have a neuro-protective M2 (alternatively-activated) phenotype and protect motor neurons, whereas microglia isolated from end-stage disease ALS mice have adopted an injurious/toxic M1 (classically-activated) phenotype and are toxic to motor neurons [23]; Astrocytes protect the BBB against hypoxia-induced disruption of tight junction protein, zonula occludens, and paracellular permeability changes by decreasing VEGF expression in porcine brain microvascular endothelial cells [24]. Thus, the transient breakdown of the BSCB may be due to compensatory mechanisms by non-neuronal cells such as astrocytes and microglia. The temporal impairment of the BSCB, in turn, seems to contribute to the degeneration of motor neurons in TDP CKO mice. Further studies to elucidate the causality between BSCB leakage and motor neuron degeneration in ALS are warranted. 


\section{Acknowledgments}

This study was supported by a Grant-in-Aid for General Scientific Research (C) from The Ministry of Education, Culture, Sports, Science and Technology (22590965 to S.S.), Grant-in-Aid for Scientific Research on Innovated Areas "Foundation of Synapse and Neurocircuit Pathology" from MEXT, Japan (M.K.) and Strategic Research Program for Brain Sciences from JST, Japan (G.S.). 


\section{References}

[1] V. Bartanusz, D. Jezova, B. Alajajian, M. Digicaylioglu, The blood-spinal cord barrier: morphology and clinical implications. Ann. Neurol. 70 (2011) 194-206.

[2] S. Garbuzova-Davis, P.R. Sanberg, Blood-CNS Barrier Impairment in ALS patients versus an animal model. Front. Cell Neurosci. Doi: 10.3389/fncel.2014.00021

[3] Y. Iguchi, M. Katsuno, J. Niwa, S. Takagi, S. Ishigaki, K. Ikenaka, K. Kawai, H. Watanabe, K. Yamanaka, R. Takahashi, H. Misawa, S. Sasaki, F. Tanaka, G. Sobue, Loss of TDP-43 causes age-dependent progressive motor neuron degeneration. Brain 136 (2013) 1371-1382.

[4] M.E. Alexianu, M. Kozovska, S.H. Appel, Immune reactivity in a mouse model of familial ALS correlates with disease progression. Neurology 57 (2001) 1282-1289.

[5] B.V. Zlokovic, The blood-brain barrier in health and chronic neurodegenerative disorders. Neuron 57 (2008) 178-201.

[6] A. Leonardi, G. Abbruzzese, L. Arata, L. Cocito, M. Vische, Cerebrospinal fluid (CSF) findings in amyotrophic lateral sclerosis. J. Neurol. 231 (1984) 75-78.

[7] P. Annunziata, N. Volpi, High levels of $\mathrm{C} 3 \mathrm{c}$ in the cerebrospinal fluid from amyotrophic lateral sclerosis patients. Acta Neurol. Scand. 72 (1985) 61-64.

[8] J. Brettschneider, A. Petzold, S.D. Süssmuth, A.C. Ludolph, H. Tumani, Axonal damage markers in cerebrospinal fluid are increased in ALS. Neurology 66 (2006) $852-856$.

[9] Z. Zhong, R. Deane, Z. Ali, M. Parisi, Y. Shapovalov, M.K. O'Banion, K. Stojanovic, A. Sagare, S. Boillee, D.W. Cleveland, B.V. Zlokovic, ALS-causing SOD1 mutants generate vascular changes prior to motor neuron degeneration. Nat. Neurosci. 11 (2008) 420-422.

[10] J.I. Engelhardt, J. Tajti, S.H. Appel, Lymphocytic infiltrates in the spinal cord in amyotrophic lateral sclerosis. Arch. Neurol. 50 (1993) 30-36. 
[11] J.S. Henkel, J.I. Engelhardt, L. Siklos, E.P. Simpson, S.H. Kim, T. Pan, J.C. Goodman, T. Siddique, D.R. Beers, S.H. Appel, Presence of dendritic cells, MCP-1, and activated microglia/macrophages in amyotrophic lateral sclerosis spinal cord tissue. Ann. Neurol. 55 (2004) 221-235.

[12] S. Garbuzova-Davis, S. Saporta, E. Haller, I. Kolomey, S.P. Bennett, H. Potter, P.R. Sanberg, Evidence of compromised blood-spinal cord barrier in early and late symptomatic SOD1 mice modeling ALS. PLoS ONE 2 (2007) e1205.

[13] C. Nicaise, D. Mitrecic, P. Demetter, R. De Decker, M. Authelet, A. Boom, R. Pochet, Impaired blood-brain and blood-spinal cord barriers in mutant SOD1-linked ALS rat. Brain Res. 1301 (2009) 152-162.

[14] R.F. Regan, Y. Guo, Toxic effect of hemoglobin on spinal cord neurons in culture. J. Neurotrauma 15 (1998) 645-653.

[15] S. Garbuzova-Davis, E. Haller, S. Saporta, I. Kolomey, S.V. Nicosia, P.R. Sanberg, Ultrastructure of blood-brain barrier and blood-spinal cord barrier in SOD1 mice modeling ALS. Brain Res. 1157 (2007) 126-137.

[16] E.A. Winkler, J.D. Sengillo, J.S. Sullivan, J.S. Henkel, S.H. Appel, B.V. Zlokovic, Blood-spinal cord barrier breakdown and pericyte reductions in amyotrophic lateral sclerosis. Acta Neuropathol. 125 (2013) 111-120.

[17] L.M. Dallasta, L.A. Pisarov, J.E. Esplen, J.V. Werley, A.V. Moses, J.A. Nelson, C.L. Achim, Blood-brain barrier tight junction disruption in human immunodeficiency virus-1 encephalitis. Am. J. Pathol. 155 (1999) 1915-1927.

[18] M.K. Luabeya, L.M. Dallasta, C.L. Achim, C.D. Pauza, R.L. Hamilton, Blood-brain barrier disruption in simian immunodeficiency virus encephalitis. Neuropathol. Appl. Neurobiol. 26 (2000) 454-462.

[19] J.S. Henkel, D.R. Beers, S. Wen, R. Bowser, A.H. Appel, Decreased mRNA expression of tight junction proteins in lumbar spinal cords of patients with ALS. Neurology 72 (2009)1614-1616. 
[20] S. Liebner, A. Fischmann, G. Rascher, F. Duffner, E.-H.Grote, H. Kakbacher, H. Wolburg, Claudin-1 and claudin-5 expression and tight junction morphology are altered in blood vessels of human glioblastomamultiforme. Acta Neuropathol. 100 (2000) 323-331.

[21] E.A. Winkler, J.D. Sengillo, R.D. Bell, J. Wang, B.V. Zlokovic, Blood-spinal cord barrier pericyte reductions contribute to increased capillary permeability. J. Cereb. Blood Flow Metab. 32 (2012) 1841-1852.

[22] Kalaria RN, Cohen DL, Premkumar DR, Nag S, LaManna JC, Lust WD. Vascular endothelial growth factor in Alzheimer's disease and experimental cerebral ischemia. Brain Res. Mol. Brain Res. 62 (1998) 101-105.

[23] Bing Liao, Weihua Zhao, David R. Beers, Jenny S. Henkel, and Stanley H. Appel. Transformation from a neuroprotective to a neurotoxic microglial phenotype in a mouse model of ALS. Exp. Neurol. 237 (2012) 147-152.

[24] Fischer S, Wobben M, Kleinstück J, Renz D, Schaper W. Effect of astroglial cells on hypoxia-induced permeability in PBMEC cells. Am. J. Physiol. Cell Physiol. 279 (2000) C935-944. 


\section{Figure legends}

Fig. 1a. The proliferation of macrophages/microglia is observed exclusively in the anterior horn of the spinal cord (arrows) (anti-MAC-2 antibody immunostaining, TDP-43 CKO mouse, 50 wks).

Fig. 1b. Activated immunoreactivity of fibrinogen is largely confined to the wall or lumen of the capillaries in the anterior horns and not spreading far into the parenchyma (TDP-43 CKO mouse, 50 wks).

Fig. 2. No reduction was detected in the expression levels of claudin-5, occludin and ZO-1 in the TDP CKO mice compared to the wild-type mice at the ages of 20, 50, and 70 wks.

Fig. 3a. The capillary consists of an endothelium (E) and a pericyte (P) (control mouse, 20 wks). The capillary is surrounded by double basement membranes (arrows).

Fig. 3b. An endothelial cell (E) is characterized by a tight junction (arrow) and relatively thin cytoplasm (control mouse, $20 \mathrm{wks}$ ).

Fig. 3c. The cytoplasm of an endothelial cell is electron-dense, showing multiple vacuoles (TDP CKO mouse, $50 \mathrm{wks}$ ).

Fig. 3d. Endothelial cells exhibiting cytoplasms diffusely swollen with edematous fluid (TDP CKO mouse, 50 wks). Pericytes (P) proliferate and circumvent around the almost whole capillary. Inset: High magnification of the square. The tight junction appears intact (arrows). 


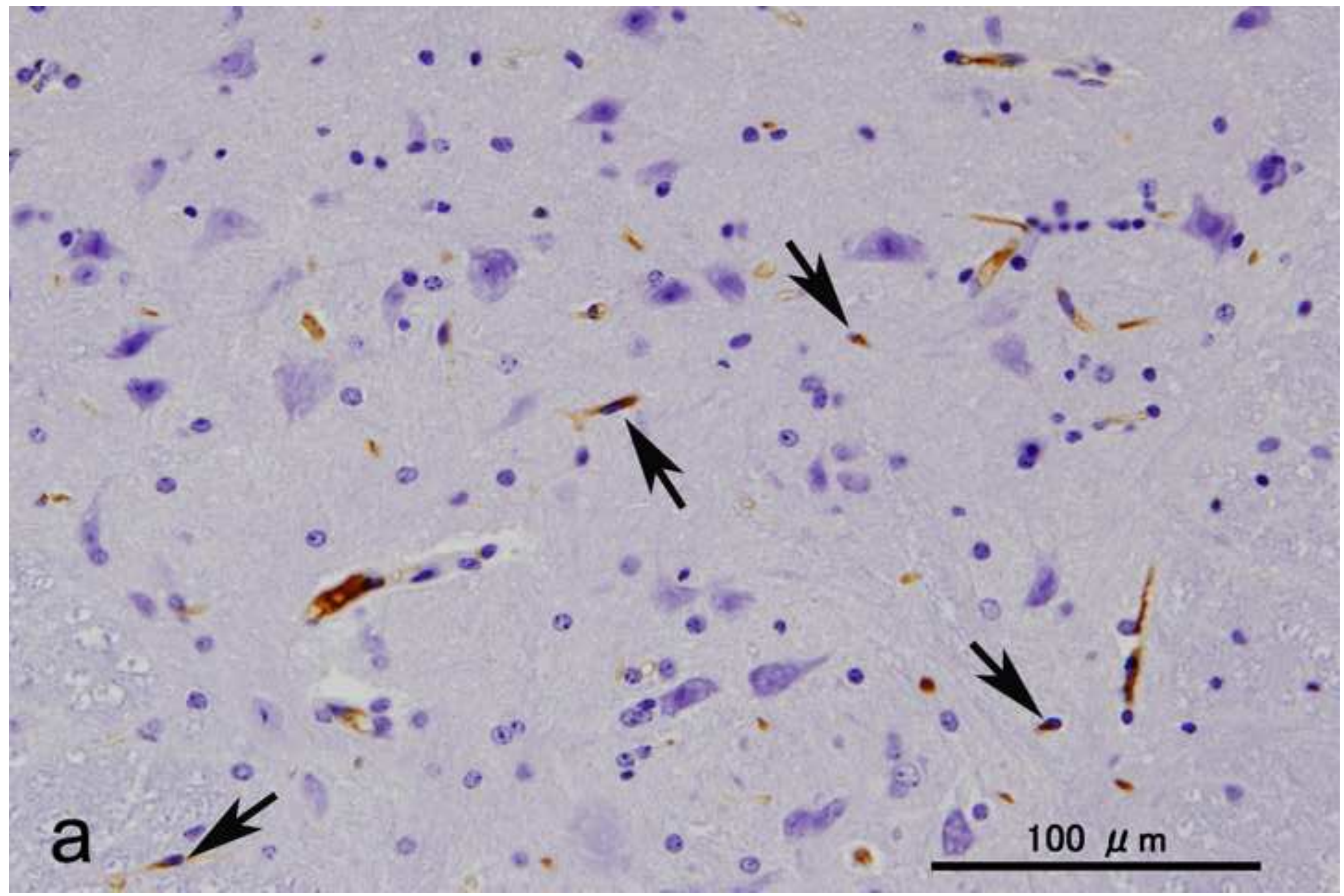




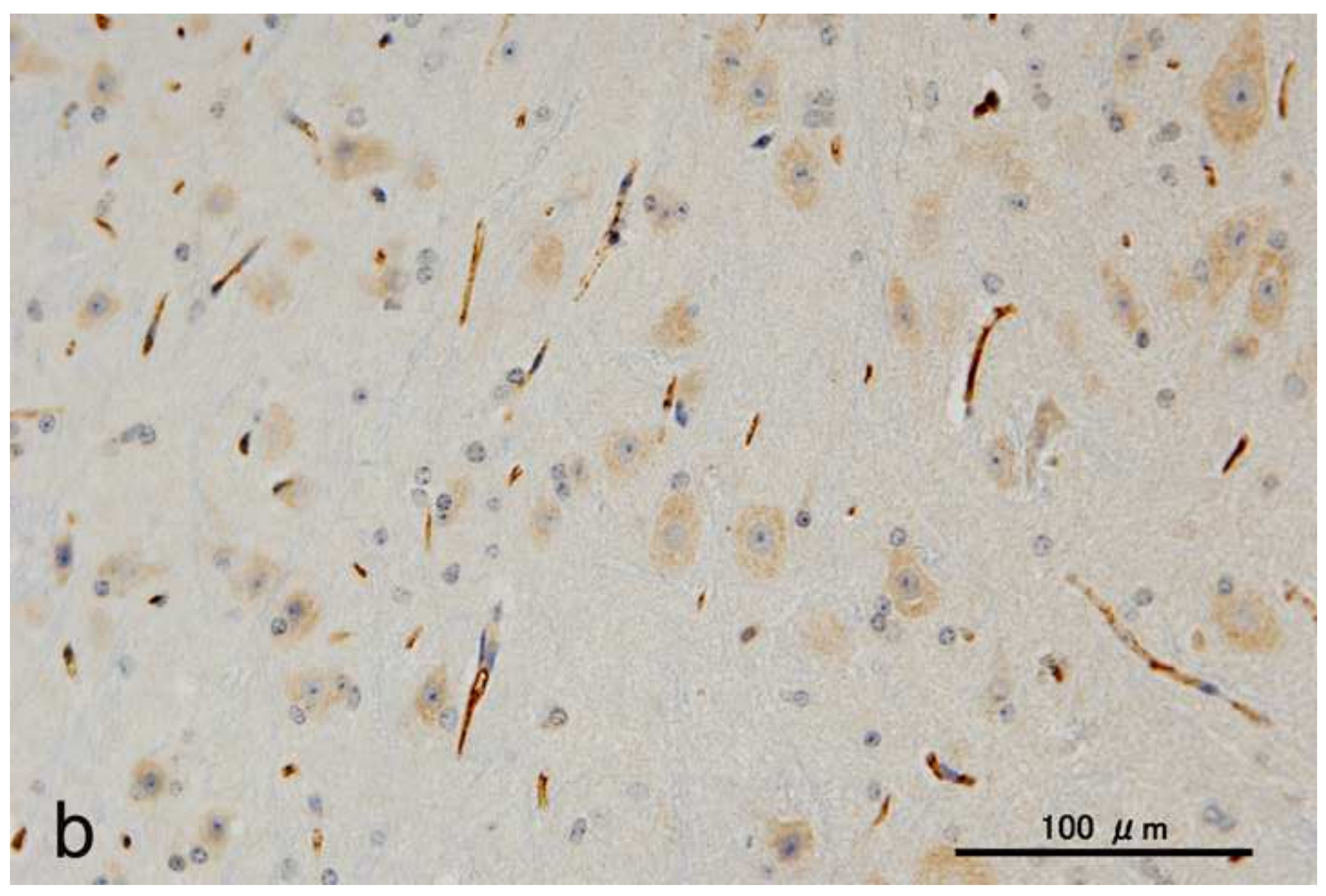


Claudin-5

$20 w$

WT

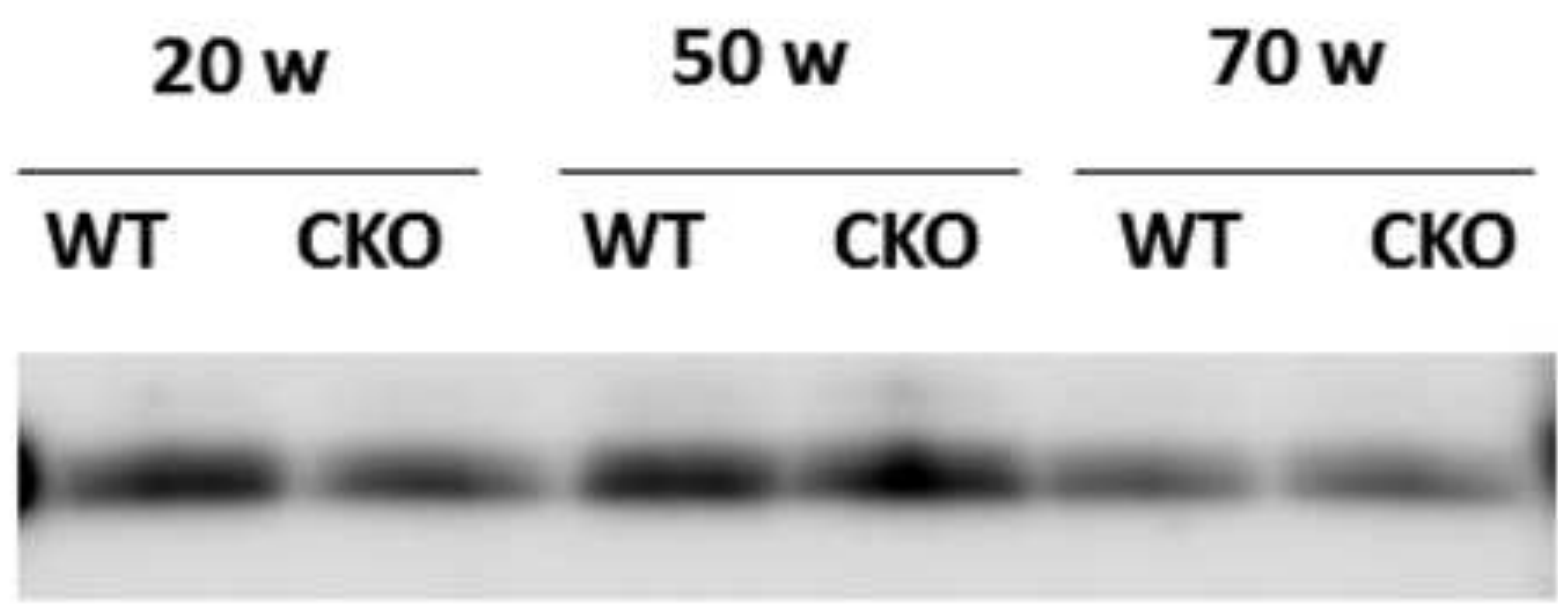

Occludin

$50 w$

$70 w$

$\begin{array}{llll}\text { CKO WT CKO WT CKO } & \end{array}$
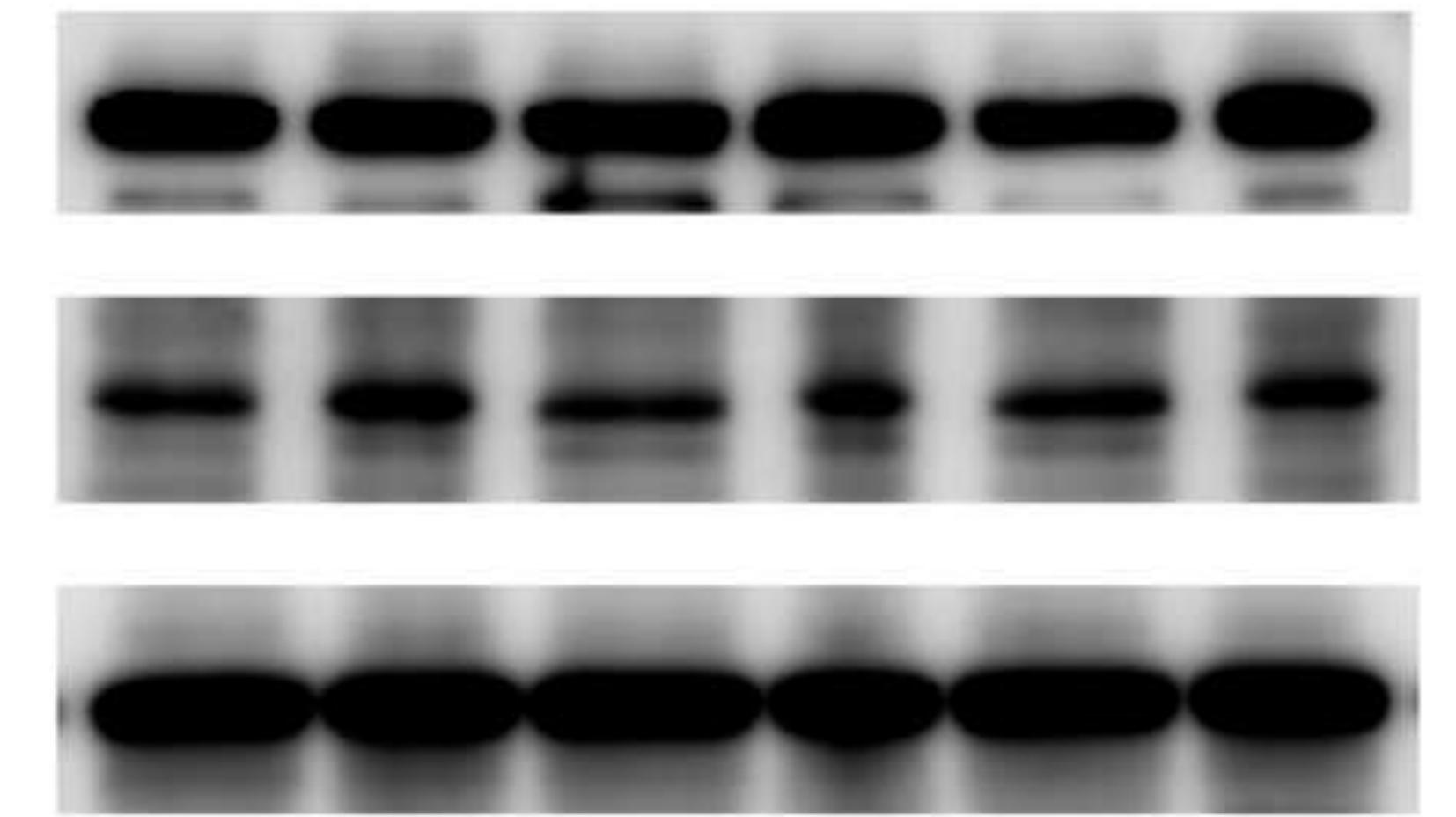

\author{
ZO-1 \\ GAPDH
}

\section{.}
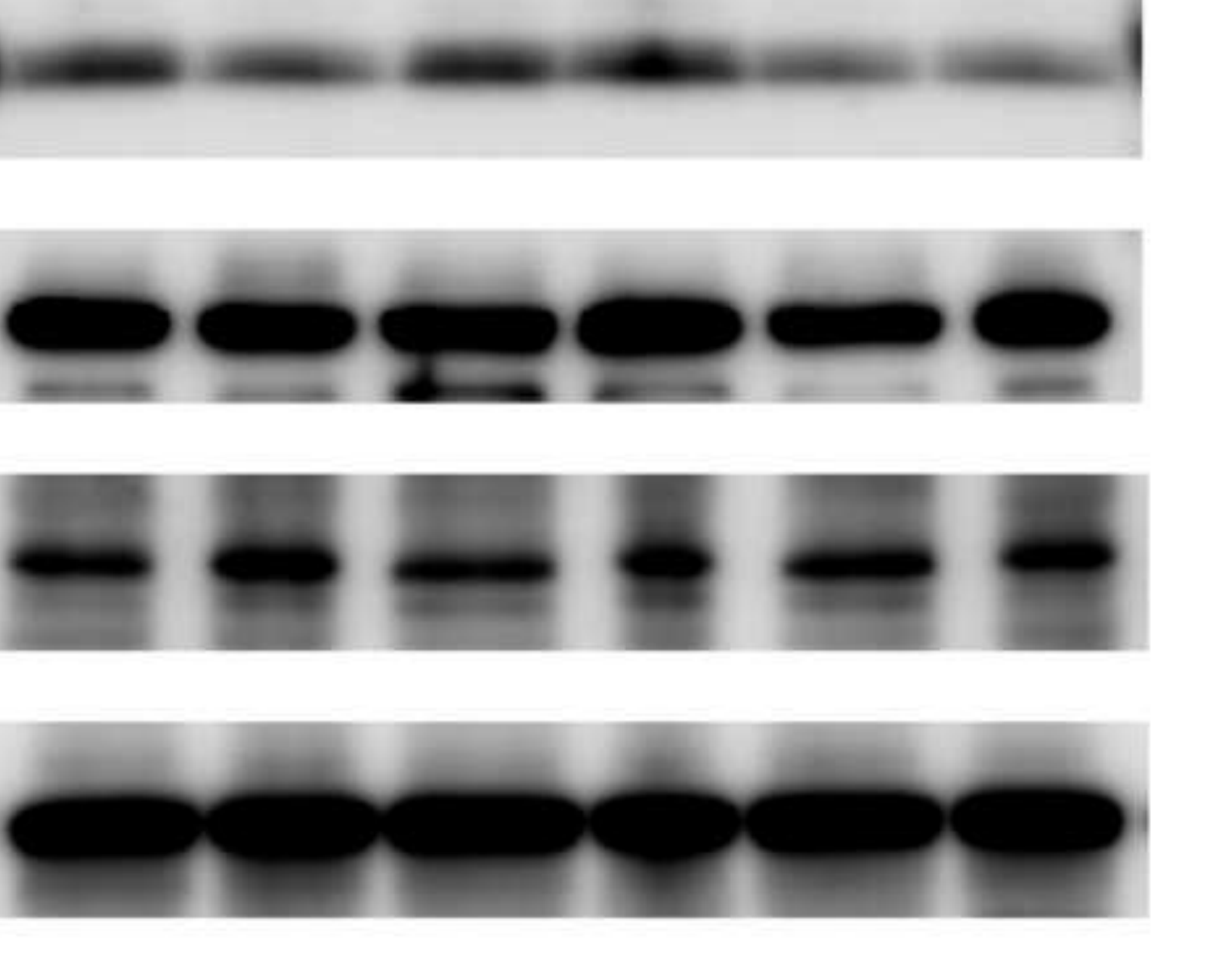


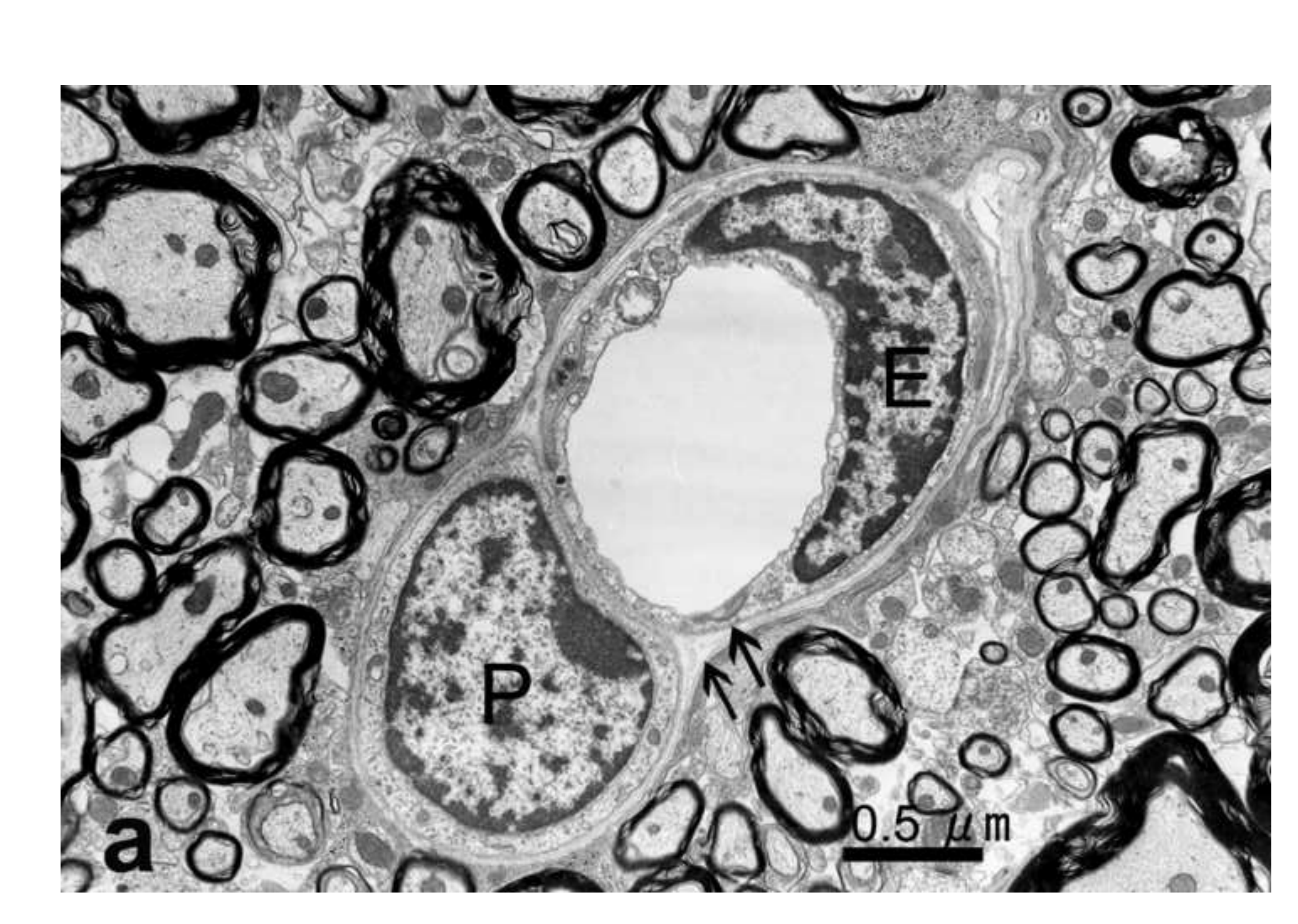




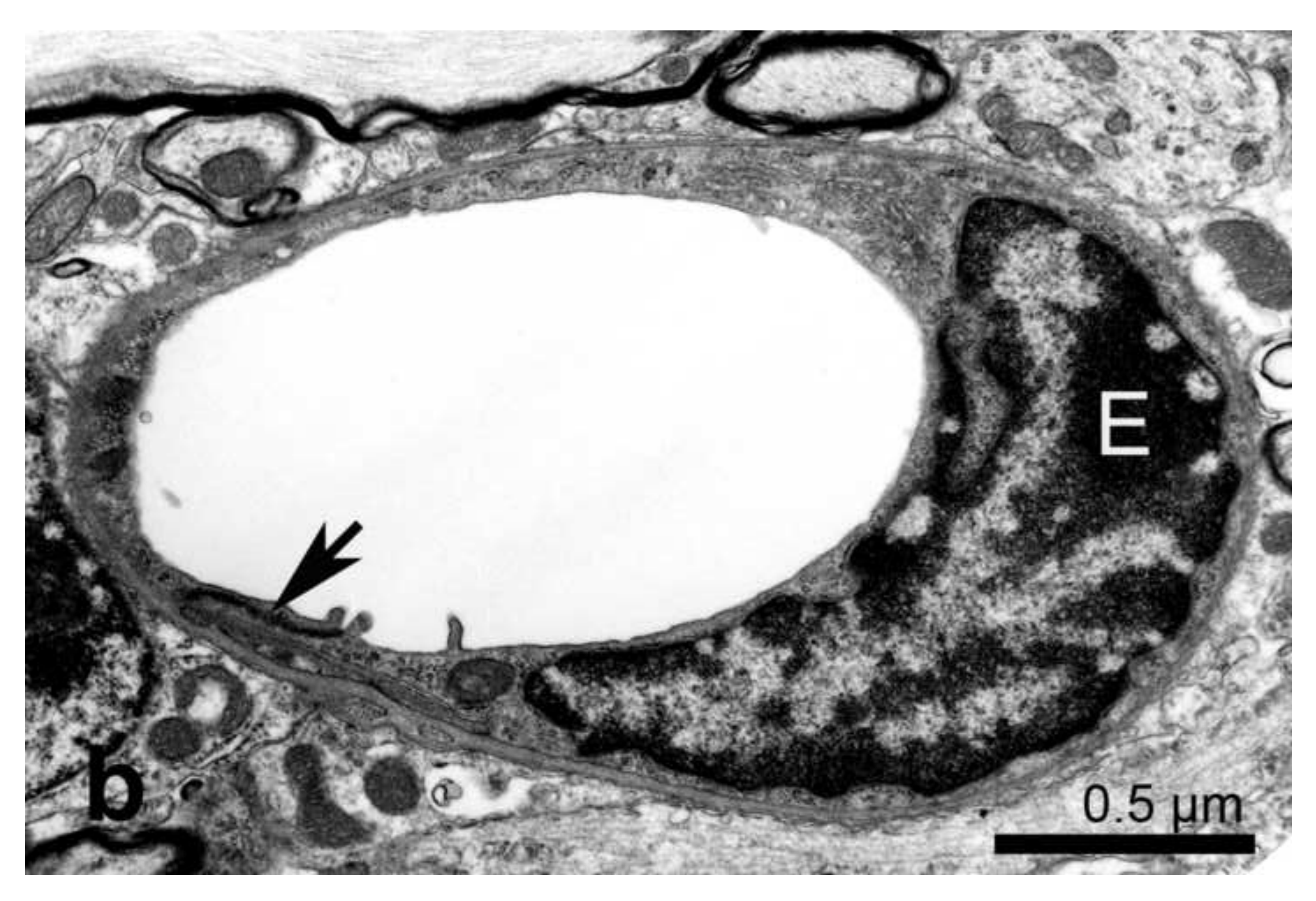

\section{-}

(

Figure 


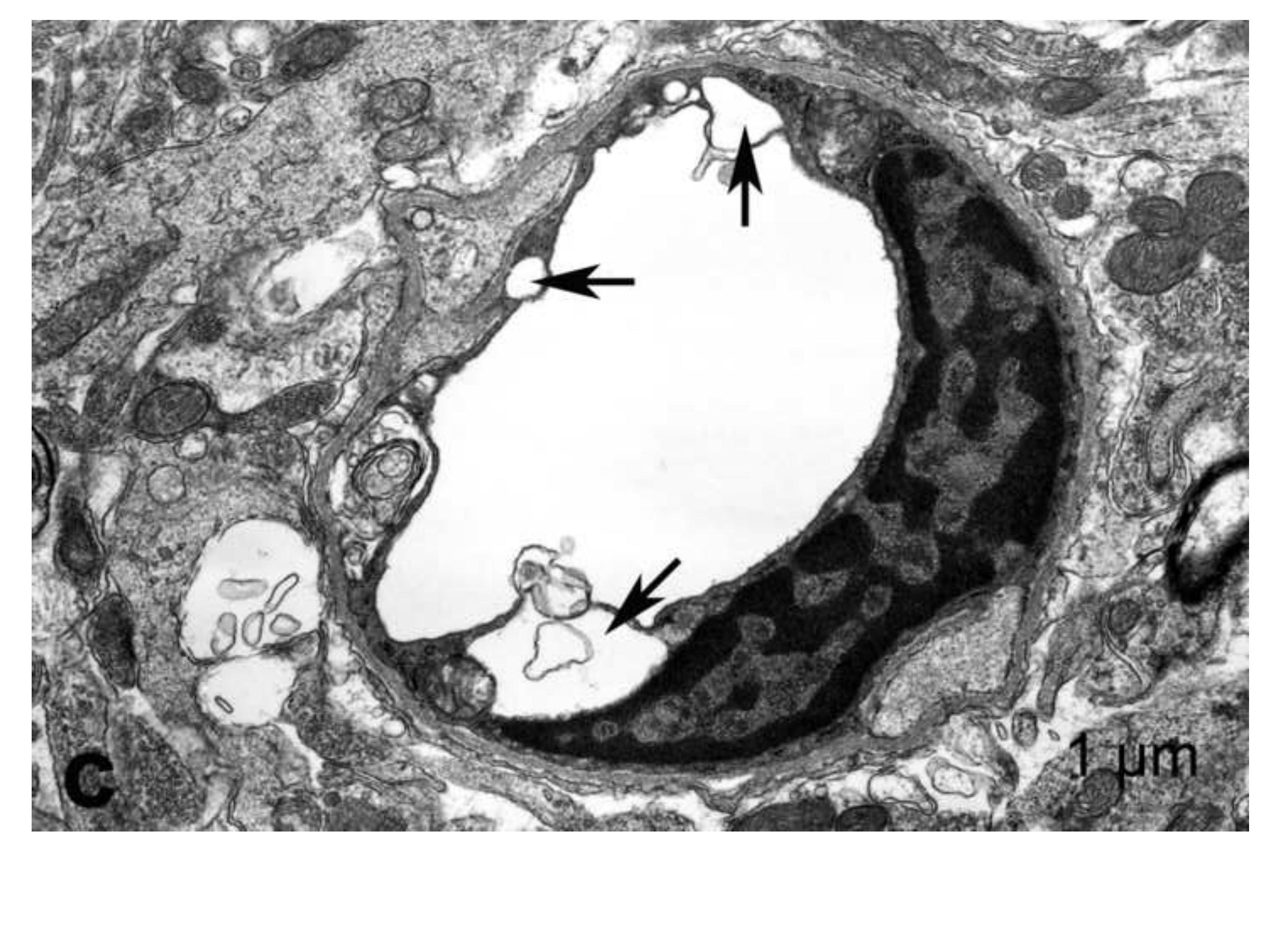

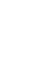



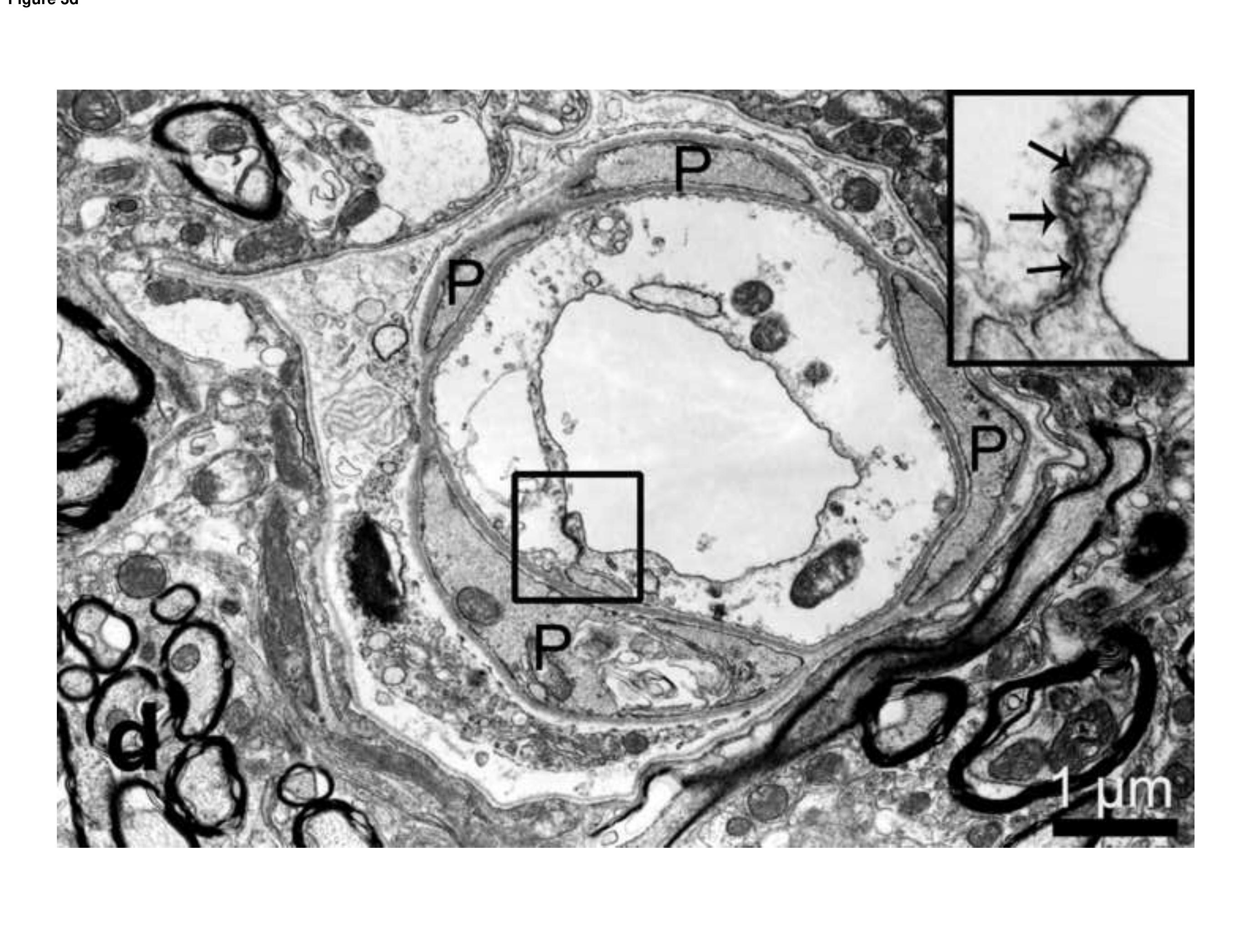